\title{
FRAGILIDADE, DESEMPENHO COGNITIVO E SINTOMAS DEPRESSIVOS DE IDOSOS RIBEIRINHOS DA AMAZÔNIA
}

Rodolfo Gomes do Nascimento

Denise da Silva Pinto

Celina Maria Colino Magalhães

Universidade Federal do Pará (UFPA)

Ronald de Oliveira Cardoso

Katiane da Costa Cunha

Universidade Estadual do Pará (UEPA)

Anna Beatriz de Souza Piedade

Giovana Rodrigues Puga

Universidade da Amazônia (UNAMA)
Recebido em: 19/07/2020

$1^{\text {a }}$ revisão em: 28/05/2021

Aceito em: 16/06/2021

\section{RESUMO}

A síndrome de fragilidade é uma condição clínica em que se observa um aumento no estado de vulnerabilidade do indivíduo envolvendo diversos fatores de ordem biopsicossociais. O objetivo foi investigar o desempenho cognitivo, sintomas depressivos e fragilidade entre idosos ribeirinhos amazônicos, bem como a associação entre essas variáveis. Trata-se de um estudo observacional do tipo transversal de abordagem quantitativa realizado no município de Cametá, Pará, Brasil. Participaram do estudo 108 idosos, a coleta de dados foi por meio do fenótipo de fragilidade postulado por Fried et al. (2001) modificado, Miniexame do Estado Mental (MEEM) e Escala de Depressão Geriátrica (EDG-15). Constatou-se que a maioria dos ribeirinhos foram classificados como idosos não frágeis, além disso, o comprometimento cognitivo e a presença de sintomas depressivos consolidaram-se como fatores associados à condição de fragilidade ( $p=0.0468 \mathrm{e}$ $p=0.0032$ ). Por fim, destaca-se a importância da gestão da fragilidade em idosos de comunidades tradicionais brasileiras.

Palavras-chave: idosos; cognição; depressão, desempenho; Amazônia. 


\title{
FRAILTY, COGNITIVE PERFORMANCE AND DEPRESSIVE SYMPTOMS OF ELDERLY PEOPLE BORDERING THE AMAZON
}

\begin{abstract}
The frailty syndrome is a clinical condition in which the individual's state of vulnerability is increased, involving several biopsychosocial factors. The aim was to investigate the study cognitive performance, depressive symptoms and frailty among elderly riverside Amazonians, as well as the association between these variables. This is an observational cross-sectional study with a quantitative approach carried out in the city of Cametá, Pará, Brazil. 108 elderly people participated in the study. Data collection was performed using the frailty phenotype postulated by Fried et al. (2001) modified, Mini Mental State Examination (MMSE) and Geriatric Depression Scale (GDS-15). It was found that most riverside dwellers were classified as non-frail elderly, in addition, cognitive impairment and the presence of depressive symptoms were consolidated as factors associated with the frail condition ( $p=0.0468$ and $p=0.0032$ ). Finally, the importance of management failty in the elderly in traditional Brazilian communities is highlighted.
\end{abstract}

Keywords: aged; cognition; depression; performance; Amazon region. 


\section{FRAGILIDAD, DESEMPEÑO COGNITIVO Y SÍNTOMAS DEPRESIVOS DE LOS ANCIANOS RIBEIRINHOS DA AMAZÔNIA}

\section{RESUMEN}

El síndrome de fragilidad es una condición clínica en la que se incrementa el estado de vulnerabilidad del individuo, involucrando varios factores biopsicosociales. El objetivo fue investigar el estudio del rendimiento cognitivo, los síntomas depresivos y la fragilidad en comunidades ancianas ribereñas de la Amazonía, así como la asociación entre estas variables. Se trata de un estudio observacional transversal con enfoque cuantitativo realizado en la ciudad de Cametá, Pará, Brasil. 108 personas mayores participaron en el estudio. La recolección de datos se realizó utilizando el fenotipo de fragilidad postulado por Fried et al. (2001) modificado, Mini Examen del Estado Mental (MMSE) y Escala de Depresión Geriátrica (GDS-15) Se encontró que la mayoría de los ribereños fueron clasificados como ancianos no frágiles, además, el deterioro cognitivo y la presencia de síntomas depresivos se consolidaron como factores asociados a la condición frágil ( $p=0.0468$ yp = 0.0032). Finalmente, se destaca la importancia de manejar em los ancianos en las comunidades brasileñas tradicionales.

Palabras clave: personas mayores; cognición; depresión; desempeño Amazonia. 


\section{INTRODUÇÃO}

Nas últimas décadas, a maioria das investigações dedicadas à compreensão da fragilidade tem revelado que o fenótipo desta síndrome é percebido como resultado de fatores predisponentes e desencadeadores múltiplos, tais como características genéticas, desenvolvimento social, hábitos de vida, padrões culturais, psiquismo, doenças em atividade e história pregressa. Desse modo, há também um crescente interesse no entendimento dos seus possíveis fatores biopsicossociais associados em idosos inseridos em diversos contextos (OforiAsenso, Chin, Mazidi, Zomer, Ilomaki, Zullo, Gazevic, Ademi, Korhonen, LoGiudice, \& Bell, 2019).

Estudos sobre o funcionamento cognitivo também tem ganhado relevância visto que os distúrbios dessa função têm sido frequentemente associados à fragilidade em idosos (Fabrício, Chagas, \& Diniz, 2020; Gifford, et al., 2019; Borges, Canevelli, Cesari, \& Aprahamian, 2019). Assim como apresentado em relação à função cognitiva, diversas pesquisas têm reforçado o conceito de confluência entre as duas condições e suas sérias repercussões no funcionamento e na qualidade de vida dos idosos (Fried, Tangen, Walston, Newman, Hirsch, Gottdiener, Seeman, Tracy, Kop, Burke, \& McBurnie, 2001; Curcio, Henao, \& Gomez, 2014; Nascimento \& Batistoni, 2019; Chu, Chang, Ho, \& Lin, 2019).

Considerando-se o impacto da síndrome de fragilidade sobre a funcionalidade e qualidade de vida dos idosos, das famílias, bem como sobre os sistemas de atenção à saúde e assistência social, revela-se um tema de interesse público, cada vez mais abordado por pesquisadores do campo gerontológico e de saúde pública em várias regiões do mundo (Araújo Júnior et al., 2019). No entanto, a maioria dos estudos realizados envolvem idosos inseridos em contextos urbanos. Considerando produção do conhecimento dessa temática em contextos rurais, nota-se que ainda é pouco conhecida, especialmente naqueles tipicamente amazônicos como as comunidades ribeirinhas, que ainda hoje representam uma importante lacuna na literatura científica.

Em meio a um contexto ecológico rico em biodiversidade e cultura, na Amazônia ribeirinha prevalece um acentuado grau de vulnerabilidade social quando comparadas às comunidades urbanas, o que reflete em graves carências em saneamento, saúde e educação (Silva, Santos, Pontes, \& Maluschke, 2011; Polaro, Gonçalves, Franhani, \& Feitosa, 2013). Vale observar que indivíduos que vivem nesses contextos tendem a apresentar piores condições de saúde, especialmente na velhice, com maior o risco de adoecimento e importante prejuízos à qualidade de vida dos idosos (Antonini, Paz, Ribeiro, Brito, Mota, Silva, Cristi-Monteiro, Jung, \& Cruz, 2016; Ribeiro, Ribeiro, Viegas, Teixeira, Santos Montagner, Mota, Barbisan, Cruz, \& Paz, 2013).

Diante desse cenário que envolve a fragilidade de idosos e a multidimensionalidade de fatores socioculturais dessas regiões, o objetivo do 
presente estudo foi investigar o desempenho cognitivo, sintomas depressivos e fragilidade entre idosos ribeirinhos amazônicos, bem como a associação entre essas variáveis.

\section{MÉTODO}

Trata-se de um estudo observacional do tipo transversal de abordagem quantitativa. Todos os preceitos éticos que regem pesquisas com seres humanos foram observados e respeitados, segundo a Resolução 466/2012 respeita a Declaração de Helsinque. A pesquisa foi aprovada pelo Comitê de Ética em Pesquisa com Seres Humanos do Núcleo de Medicina Tropical da Universidade Federal do Pará (CEP/NMT/UFPA), segundo o parecer de n 926.744/2014.

O estudo foi realizado na região das ilhas do município de Cametá, Pará, Brasil, estando situado no nordeste paraense, distante em linha reta $156 \mathrm{~km}$ da capital do estado, Belém. É um município eminentemente ribeirinho, envolvendo geograficamente cerca de 100 ilhas fluviais potencialmente habitáveis.

A amostra da pesquisa foi constituída por 108 idosos. Para o cálculo do tamanho mínimo da amostra, considerou-se a estimativa da proporção populacional de idosos residentes na região das ilhas do município, limites de confiança de $10 \%$ e nível $\alpha$ de confiança de $90 \%$. Para a seleção dos idosos, adotou-se os seguintes critérios de inclusão: ser residente em comunidades ribeirinhas tradicionais em situação de isolamento territorial; e que aceitasse participar voluntariamente da pesquisa por meio da assinatura do Termo de Consentimento Livre e Esclarecido.

Na intenção de manter uma uniformização na linha de pesquisas sobre fragilidade, foram adotados os mesmos critérios de exclusão postulados por Fried et al. (2001) e Ferrucci, Guralnik, Studenski, Fried, Cutler Junior e Walston (2004). Dessa forma, os critérios de exclusão foram: apresentar incapacidade permanente ou temporária para andar, exceto com uso de dispositivo de auxílio à marcha; apresentar perda localizada de força e afasia decorrentes de sequela de acidente vascular encefálico; apresentar comprometimento grave da motricidade, da fala ou da afetividade associados à doença de Parkinson avançada; possuir alterações auditivas ou visuais severas e incapacitantes para a realização dos testes propostos e para a manutenção do diálogo adequado aos questionamentos durante a coleta de dados; e ser cadeirante, acamado ou estar em estágio terminal de alguma doença.

A avaliação foi realizada por dois pesquisadores, previamente treinados e habituados ao contexto de investigação. Cabe a ressalva que o período de habituação dos pesquisadores ocorreu ao longo de aproximadamente dois anos, por meio de viagens sucessivas às ilhas no sentido de integrá-los ao ambiente e às comunidades ribeirinhas. A coleta de dados ocorreu em local apropriado, durante uma sessão, de forma individual, com duração média de 60 minutos. Os instrumentos utilizados foram: questionário sociodemográfico, modelo do fenótipo de fragilidade, Mini Exame do Estado Mental (MEEM) e a versão reduzida 
da Escala de Depressão Geriátrica (EDG-15). O questionário sociodemográfico foi previamente elaborado pelos pesquisadores contendo as seguintes variáveis: sexo, idade, escolaridade, estado civil, renda pessoal e arranjo domiciliar.

No delineamento da operacionalização da síndrome de fragilidade foi utilizado o modelo postulado por Fried et al. (2001) modificado, composto por cinco domínios, a saber: a) perda de peso não intencional, b) sensação de exaustão ou fadiga autorreferida, c) baixa força de preensão manual, d) atividade física insuficiente e, e) baixa velocidade da marcha. Os idosos participantes do estudo foram classificados como frágeis se três, quatro ou cinco itens estivessem presentes; pré-frágeis, se um ou dois itens estivessem presentes; ou não-frágeis, se nenhum critério estivesse presente.

Nesta investigação, para avaliar o domínio "atividade física insuficiente" foi utilizado o Questionário de nível de atividade física para idosos validado por Rauchbach et al. (2019) e modificado pelos autores. Optou-se pela utilização desse questionário por considerá-lo mais próximo da realidade dos idosos brasileiros e pela facilidade de aplicação. Devido às particularidades do contexto ribeirinho foram necessárias adaptações no conteúdo para contemplar as prováveis atividades rotineiras desempenhadas pelos idosos nesses ambientes.

O instrumento MEEM possibilitou o rastreio de possível comprometimento cognitivo. É um instrumento amplamente utilizado em todo o mundo e de fácil aplicação; envolve categorias de resposta verbais e não verbais e sua estrutura é composto por diversas questões tipicamente agrupadas em sete categorias, cada uma delas desenhada com o objetivo de avaliar funções cognitivas especificas: orientação no tempo, orientação no espaço, registro, memória de evocação, linguagem, e atenção e cálculo (Bertolucci, Brucki, Campacci, \& Juliano, 1994; Brucki, Nitrini, Caramelli, Bertolucci, \& Okamoto, 2003). Este instrumento é fortemente influenciado pelo nível de escolaridade do indivíduo avaliado e que as características culturais podem alterar o desempenho no teste. Desse modo, considerando a heterogeneidade cultural amazônica e a alta prevalência de idosos com baixa escolaridade nas comunidades ribeirinhas, foram adotadas as notas de corte recomendadas por Bertolucci et al. (1994): 13 pontos para idosos analfabetos, 18 para idosos com ensino fundamental e 26 para os idosos com ensino médio e superior.

Para a avaliação da presença de sintomas depressivos foi utilizada a Escala de Depressão Geriátrica, na sua versão reduzida (EDG-15). É um instrumento recomendado pela Organização Mundial de Saúde (OMS) e o mais empregado para este fim em populações geriátricas. Foi traduzido para o português e adaptado para aplicação no Brasil por Stoppe Júnior, Jacob Filho e Louza Neto (1994). Nesta pesquisa, adotou-se o ponto de corte 5/6 como não caso/caso para avaliação dos sintomas depressivos, recomendado por Almeida e Almeida (1999). Portanto, valores abaixo de seis foram considerados normais e valores acima ou iguais a seis foram considerados indicativos de sintomas depressivos. 
Quanto à análise dos dados, foi realizada nos programas estatísticos Epi Info versão 7.1 e o BioEstat versão 5.0, a partir da estatística descritiva através da distribuição da contagem de frequências simples e percentuais. As diferenças encontradas foram testadas pelo teste Qui - quadrado de Pearson ou, nas amostras pequenas, pelo teste $G$ de independência com a correção de Williams. Para investigar a associação entre as variáveis explicativas com a prevalência de fragilidade biológica foram usados os testes Qui - quadrado de Pearson e teste $G$ de independência com a correção de Williams; as razões de prevalência bruta e ajustada por odds ratio. Para melhor ajuste estatístico, a variável resposta foi distribuída de forma dicotômica em: 1) classificações frágil e pré-frágil e 2) classificação não-frágil.

\section{RESULTADOS}

Em relação à amostra geral $(n=108)$, a média de idade foi de 70 anos e 4 meses (desvio padrão de $\pm 7,64$ anos), sendo $50,9 \%$ ( $n=55$ ) de idosos do sexo masculino, $74,1 \%(n=80)$ casados e 49,1\% ( $n=53$ ) com convivência domiciliar com cônjuges e descendentes. Tendo em vista os aspectos socioeconômicos, evidenciou-se que a maioria era de indivíduos analfabetos funcionais, 59,3\% $(n=64)$ da amostra tinha o ensino fundamental incompleto e $65,7 \%(n=71)$ tinha renda pessoal de até um salário-mínimo.

Conforme o modelo de investigação adotado neste estudo para a identificação da fragilidade biológica, a prevalência desta síndrome entre os idosos ribeirinhos foi de $9,3 \%$. Do total da amostra, a condição de pré-fragilidade estava presente em $38,9 \%$ dos casos e $51,9 \%$ foram considerados idosos não-frágeis.

Em relação ao desempenho cognitivo, dos 108 idosos investigados 9,3\% apresentavam comprometimento cognitivo com média de pontuação de $26,94 \pm 2,4$ pontos. Sobre o rastreio de sintomas depressivos, $13 \%$ preencheram os critérios sugestivos de transtorno de humor com uma média de 2,69 2,42 pontos. Não houve diferença estatisticamente significativa na análise entre os sexos para ambas as investigações ( $p=0,1102$ e 0,0743) (Tabela 1).

Tabela 1.

Comprometimento cognitivo dos idosos ribeirinhos conforme o sexo.

Cametá, Pará, 2017.

\begin{tabular}{|c|c|c|c|c|c|c|c|}
\hline Variável & \multicolumn{2}{|c|}{ Total } & \multicolumn{2}{|c|}{ Masculino } & \multicolumn{2}{|c|}{ Feminino } & \multirow[t]{2}{*}{ valor-p } \\
\hline & $\mathbf{n}$ & $\%$ & $\mathbf{n}$ & $\%$ & $\mathbf{n}$ & $\%$ & \\
\hline \multicolumn{8}{|c|}{ Comprometimento cognitivo } \\
\hline Sim & 10 & 9,3 & 7 & 12,7 & 3 & 5,7 & 0.2102 \\
\hline Não & 98 & 90,7 & 48 & 87,3 & 50 & 94,3 & \\
\hline \multicolumn{8}{|c|}{ Sintomas depressivos } \\
\hline Sim & 14 & 13 & 4 & 7,3 & 10 & 18,9 & 0,0743 \\
\hline Não & 94 & 87 & 51 & 92,7 & 43 & 81,1 & \\
\hline
\end{tabular}


A despeito do rastreio pelo MEEM, ao analisar o desempenho nos subitens do teste com a finalidade de identificar a prevalência do comprometimento das funções cognitivas específicas, observou-se que $89,8 \%$ dos idosos não conseguiram realizar os cálculos propostos para avaliar a função "Atenção e cálculo". Essa foi a pior média de acerto do teste $(1,34 \pm 1,69)$, cuja pontuação do domínio varia de 0 a 5 . Outros itens avaliados que também demandam de educação formal seguiram o mesmo comportamento de baixo desempenho. São exemplos disso, o item "Escrita de uma frase completa" com 77,8\% e o item "Ler/Executar", que avalia a leitura e compreensão, com 63,9\%.

Os resultados revelaram que o melhor desempenho foi do item "Registro" que avalia a memória imediata. Dos 108 idosos avaliados, 92,6\% conseguiram repetir acertadamente as três palavras ditas pelo avaliador. No entanto, quando avaliada a memória de trabalho pelo item "Evocação das palavras", a proporção de acerto das três palavras foi de 44,4\% com média de 1,92 $\pm 1,15$.

Outros achados foram os referentes à orientação espaço-temporal dos idosos ribeirinhos, na qual a pontuação varia de 0 a 5 , constatou-se uma média de acertos de $4,17 \pm 1,31$. Do total, cerca de $61 \%$ dos idosos conseguiram acertar todos os itens questionados. Nesse domínio, o item com maior destaque quanto à porcentagem de erro foi o "ano" com 28,7\%. De forma bem similar, quando avaliado o desempenho nos itens relativos à "Orientação espacial", verificou-se uma média de acertos de 4,45 \pm 0,89. Dos 108 idosos avaliados, $63 \%$ deles acertaram todos os itens, sendo que o item que conferiu maior percentual de erro foi o "Estado" com 33,3\%.

Finalmente as associações entre a variável de desfecho (condições frágeis e préfrágil agrupadas) e as variáreis independentes (comprometimento cognitivo e sintomas depressivos) mostrou-se estatisticamente significativa expressa em $p=$ 0.0468 e $p=0.0032$, respectivamente (Tabela 2 ).

Tabela 2.

Razão de chance (OR) bruta e intervalo de confiança (IC) para fragilidade segundo comprometimento cognitivo e sintomas depressivos. $(\mathrm{N}=108)$. Cametá, Pará, 2017.

\begin{tabular}{|c|c|c|c|c|c|c|c|}
\hline \multirow{2}{*}{ Variáveis } & \multirow{2}{*}{$\mathbf{N}$} & \multicolumn{2}{|c|}{ Fragilidade } & \multirow{2}{*}{$\begin{array}{c}\text { valor- } \\
p \\
\end{array}$} & \multirow{2}{*}{ OR } & \multicolumn{2}{|c|}{$1 \mathrm{C} 95 \%$} \\
\hline & & n & Prevalência & & & Inferior & Superior \\
\hline \multicolumn{8}{|c|}{$\begin{array}{l}\text { Comprometimento } \\
\text { cognitivo }\end{array}$} \\
\hline Sim & 10 & 8 & $80,00 \%$ & $0.0468^{*}$ & 4,91 & 0,99 & 24,31 \\
\hline Não & 98 & 44 & $44,90 \%$ & & & & \\
\hline \multicolumn{8}{|l|}{$\begin{array}{l}\text { Sintomas } \\
\text { depressivos }\end{array}$} \\
\hline Sim & 14 & 12 & $85,71 \%$ & $0.0032^{*}$ & 0,12 & 0,03 & 0,58 \\
\hline Não & 94 & 40 & $42,55 \%$ & & & & \\
\hline
\end{tabular}




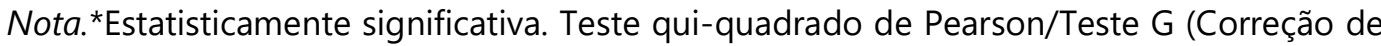
Williams).

\section{DISCUSSÃO}

Neste estudo, houve preponderância de indivíduos que compartilham um perfil sociodemográfico de idosos mais jovens, casados, com baixa escolaridade e renda, dados que corroboram tanto com a literatura já publicada sobre fragilidade de idosos de contextos rurais (Llano, Lange, Sequeira, Jardim, Castro, \& Santos, 2019; Preto, Conceição, Amaral, Figueiredo \& Preto, 2018), quanto a que se dedica a investigar condições de saúde de idosos ribeirinhos (Ribeiro et al., 2013; Polaro et al., 2013; Carneiro \& Magalhães, 2020). Vale destacar que em contextos socioeconômicos desfavoráveis, tal qual o rural e o ribeirinho, fatores como baixa renda e escolaridade podem implicar no estilo de vida e nas condições de saúde da população idosa, podendo ser desfecho para a fragilidade. Assim, quanto maior a vulnerabilidade social, maiores serão os prejuízos à qualidade de vida e ao bemestar dos idosos, fator este que pode influenciar ou desencadear o processo de fragilização do idoso (Gutierrez-Robledo \& Ávila-Funes, 2012; Jesus, Ortolandi, Grazziano \& Zazzetta, 2017).

Em relação à fragilidade, a maioria dos idosos ribeirinhos investigados se apresentou não-frágil (51,9\%), seguido de pré-frágil (38,9\%) e frágil $(9,3 \%)$. Na literatura, existe uma ampla variabilidade de prevalência de fragilidade biológica. Resguardadas as possibilidades de comparações desses dados com outros estudos nacionais já realizados em contextos rurais e em situação de vulnerabilidade social, observa-se que os padrões de fragilidade foram menos prevalentes entre os ribeirinhos. Um estudo com idosos moradores de regiões em situação de vulnerabilidade social do município de Lafaiete Coutinho (BA) evidenciou elevadas prevalências de fragilidade, 23,8\% apresentaram prevalência de fragilidade e 57,8\% eram pré-frágeis (Reis Junior, Carneiro, Coqueiro, Santos, \& Fernandes, 2014). Outro estudo, realizado em uma população de idosos socialmente vulneráveis no contexto da pobreza de São Carlos (SP) identificou 37,7\% de idosos frágeis e 21,2\% de pré-frágeis (Jesus, Diniz, Lanzotti, Orlandi, Pavarin, \& Zazzetta, 2018). Uma pesquisa com idosos da zona rural de Pelotas (RS), os autores obtiveram 43,4\% de fragilidade e $37,1 \%(n=304)$ de pré-fragilidade (Llano et al., 2019).

As divergências encontradas em relação à ocorrência de fragilidade podem ser explicadas pela inexistência de uma definição consensual sobre a operacionalização desse constructo, uma vez que embora a maioria opte pelos parâmetros de fragilidade propostos por Fried, outros autores preferem aplicar escalas autorreferidas, com uso de todas as dimensões ou apenas algumas delas que avaliam domínios do humor, cognição e apoio social (Araújo Junior, Machado, Santos-Orlandi, Pergola-Marconato, Pavarini, \& Zazzetta, 2019). Somado a isso, deve-se considerar as distintas características ambientais e o modo de vida no contexto ribeirinho que podem influenciar positivamente no processo de 
envelhecimento e funcionando, portanto como fator de proteção à fragilização, como estilo de vida ativo fisicamente e o extenso suporte social, típico de comunidades tradicionais (Ribeiro et al., 2013; Nascimento, Cardoso, Santos, Pinto, \& Magalhães, 2016; Nascimento, Cardoso, Santos, Pinto, \& Magalhães, 2017; Freire Junior, Fernandes, Borges, Guerra, \& Abreu, 2018).

Em relação aos rastreios do desempenho cognitivo e de humor/comportamento, apenas 9,3\% dos idosos ribeirinhos apresentavam algum declínio cognitivo e 13\% foram classificados com indicativo de sintomas depressivos, prevalências inferiores ao do estudo FIBRA-BR (24,8\% e 21,4\%, respectivamente), a primeira investigação multicêntrica e de abrangência nacional de fragilidade biológica (Neri, Yassuda, Araújo, Eulálio, Cabral, Siqueira, Santos, \& Moura 2013).

Estudos já realizados com idosos rurais da região amazônica como o de Costa, Leão e Campos (2020) e de Oliveira, Pereira, Prestes, Silva e Campos (2020) revelaram importantes déficits cognitivos. No entanto, cabem algumas ressalvas. Do ponto de vista operacional, os autores utilizaram maiores escores medianos (Bruck et al., 2003), inviabilizando, portanto, comparações com o presente estudo. Além disso, de modo comparativo ao meio rural de terra firme, o contexto ruralribeirinho, ao qual se refere esta investigação, apresenta características diferenciadas no que diz respeito ao modo de vida, que é essencialmente regido pela natureza e tradições culturais particulares transmitidas ao longo das gerações.

Os dados evidenciados neste estudo sobre cognição e humor ratificam a compreensão dinâmica e multifatorial das condições de saúde no envelhecimento, inclusive em contextos rurais diferenciados. Pode-se denotar que embora haja importantes vulnerabilidades sociais, os idosos parecem não apresentar declínio cognitivo e sintomas depressivos. Costa, Rodrigues, Santos, Teixeira e Lira (2021) ao investigarem a qualidade de vida de idosos ribeirinhos, identificaram melhores resultados nos aspectos psicológico, relações sociais e meio ambiente. De acordo com alguns autores, existem importantes características do contexto ruralribeirinho amazônico que se sobrepõem às precárias condições de acesso à saúde isolamento territorial como, por exemplo, o ambiente de moradia mais pacato e tranquilo, com menor poluição; o estilo de vida mais ativo, em razão das atividades cotidianas desenvolvidas ao longo da vida; o estreitamento dos vínculos sociais e familiares, dada a típica formação das comunidades, dentre outros fatores que geram bem-estar e que são fortemente influenciados por variáveis sociocognitivas e culturais (Nascimento et al., 2017; Ribeiro et al., 2013; Costa et al., 2021).

Particularmente, com relação ao baixo desempenho dos idosos nos testes em que se avaliam as funções "atenção e cálculo" e "leitura e compreensão", percebe-se claramente a influência do nível de instrução dos idosos nessas comunidades. Assim, deve-se considerar que as porcentagens de erro podem ter sido motivadas por fatores relacionados à baixa escolaridade formal e por fatores culturais, tal qual acontece em populações rurais (Tavares et al., 2017; Costa et al., 2020). 
Sobre essa investigação cabem algumas considerações metodológicas que foram construídas ao longo do planejamento e desenvolvimento desta pesquisa em função das particularidades da amostra e do contexto ribeirinho. Considerando o fato de que a formação e a cultura poderiam influenciar as respostas e que, portanto, alguns termos utilizados no MEEM original não seriam bem compreendidos foi necessária a adequação do instrumento às especificidades linguísticas e culturais ribeirinhas preservando as intenções da versão original. Por exemplo, o uso de "localidade/ilha" para a orientação espacial em vez de "bairro", o uso do termo "diminuir" em vez de "subtrair" para a atenção e cálculo, o uso do termo "quebrar" em vez de "dobrar" para o comando de estágios, uma vez que esta é uma expressão de maior familiaridade nas regiões investigadas. BritoMarques e Cabral-Filho (2005), comentam que há evidências crescentes de que a educação e a cultura afetam o teste do estado cognitivo em diferentes grupos culturais. Desse modo é importante adaptar/adequar o MEEM a populações idosas brasileiras de baixa escolaridade e inseridas em contextos culturais diferenciados ao urbano.

É importante ressaltar que a presença de comprometimento cognitivo apresentou forte associação com a condição de fragilidade, de maneira semelhante a outros estudos nacionais com idosos inseridos em contexto rural (Llano et al., 2019; Soares, Ramos, \& Pinto, 2018). A associação entre fragilidade biológica e alteração cognitiva ainda é bastante discutida na literatura. Muitos pesquisadores reforçam a ideia de que a fragilidade pode ser preditora de transtorno cognitivo leve, de demência e de declínio cognitivo ao longo do tempo, no entanto, como tanto a fragilidade física quanto o comprometimento cognitivo têm mecanismos fisiopatológicos em comum, é difícil estabelecer uma proporção definida na relação causa-efeito (Miyamura et al., 2019; Robertson, Savva, Coen, \& Kenny, 2014).

Foi possível inferir associação entre a presença de sintomas depressivos com a condição de fragilidade dos idosos ribeirinhos. Essa associação faz sentido, uma vez que a "exaustão", um dos critérios de fragilidade, faz parte dos critérios de diagnóstico para depressão. Assim, como apresentado em relação à função cognitiva, algumas pesquisas realizadas em contexto rural ou pobreza também tem mostrado a associação positiva entre a presença de sintomas depressivos e fragilidade biológica (Curcio et al., 2014; Zazzetta et al., 2017).

A crescente tendência de associação entre sintomas depressivos e condições de fragilidade entre idosos pode estar vinculada à sobreposição de características coexistentes em tais condições de saúde, como a inatividade, a exaustão e a redução de atividades físicas. É possível que os sintomas de depressão levem a situações de fragilidade biológica e vice-versa em decorrência do compartilhamento de mecanismos fisiopatológicos e biomarcadores (Vaughan, Corbin, \& Goveas, 2015; Nascimento \& Batistoni, 2019). Sendo assim, para determinar a relação preditiva entre essas condições são necessárias outras 
estratégias metodológicas como, por exemplo, estudos longitudinais prospectivos (Mezuk, Lohman, Dumenci, \& Lapane, 2013).

Esta investigação apresenta algumas limitações a serem preenchidas em pesquisas futuras sobre a temática. Por se tratar de uma amostragem não probabilística por conveniência, o estudo não permitiu a generalização dos resultados para todos os idosos residentes em regiões ribeirinhas da Amazônia, além disso, o desenho transversal adotado não permite o estabelecimento de relação de causa e efeito. Contudo, as evidências alcançadas nesta investigação podem contribuir para aprofundar o conhecimento das condições de saúde biopsicossocial entre idosos de regiões ribeirinhas amazônicas e fornecer subsídios para o planejamento e implementação de intervenções e ações de cuidado direcionadas a essa população.

\section{CONSIDERAÇÕES FINAIS}

O presente estudo identificou que a maioria dos idosos ribeirinhos eram nãofrágeis com baixas prevalências de comprometimento cognitivo e de sintomas depressivos. Com isso, entende-se que o conhecimento revelado até o momento, nos permite inferir que embora se perceba um importante contexto de vulnerabilidade social, a maioria dos idosos investigados experienciam a fase da velhice com vigor físico e sem graves déficits cognitivos e de humor, apesar de achados adversos terem sido relatados em outros estudos. Por fim, considerando a gestão da fragilidade, importante meta gerontológica em saúde pública, destacase a importância do diagnóstico de fatores associados à essa condição em idosos de comunidades tradicionais brasileiras.

\section{REFERÊNCIAS}

Almeida, O. P., \& Almeida, S. A. (1999). Confiabilidade da versão brasileira da Escala de Depressão em Geriatria (GDS) versão reduzida. Arquivos de Neuro-psiquiatria, 57(2B), 421-426. https://doi.org/10.1590/S0004-282X1999000300013

Antonini, T. C., Paz, J. A., Ribeiro, E. E., Brito, E., Mota, K. S., Silva, T. L., ... \& Cruz, I. (2016). Impact of functional determinants on 5.5-year mortality in Amazon riparian elderly. Revista Panamericana de Salud Pública, 40, 9-15.

Araújo Júnior, F. B., Machado, I. T. J., Santos-Orlandi, A. A., Pergola-Marconato, A. M., Pavarini, S. C. I., \& Zazzetta, M. S. (2019). Fragilidade, perfil e cognição de idosos residentes em área de alta vulnerabilidade social. Ciência \& Saúde Coletiva, 24(8), 3047-3055. https://doi.org/10.1590/1413-81232018248.26412017

Bertolucci, P. H. F., Brucki, S. M. D., Campacci, S. R., \& Juliano, Y. (1994). O Mini-Exame do Estado Mental em uma população geral: impacto da escolaridade. Arquivos de Neuro-psiquiatria, 52(1), 1-7. https://doi.org/10.1590/S0004-282X1994000100001

Borges, M. K., Canevelli, M., Cesari, M., \& Aprahamian, I. (2019). Frailty as a predictor of cognitive disorders: a systematic review and meta-analysis. Frontiers in medicine, 6, 26. https://dx.doi.org/10.3389\%2Ffmed.2019.00026

Brito-Marques, P. R. D., \& Cabral-Filho, J. E. (2005). Influence of age and scholing on the performance in a modified Mini-Mental State Examination version: a study in Brazil Northeast. Arquivos de Neuro-psiquiatria, 63(3A), 583-587. https://doi.org/10.1590/S0004-282X2005000400005 
Brucki, S., Nitrini, R., Caramelli, P., Bertolucci, P. H., \& Okamoto, I. H. (2003). Sugestões para o uso do mini-exame do estado mental no Brasil. Arquivos de Neuro-psiquiatria, 61(3B), 777-781. https://doi.org/10.1590/S0004-282X2003000500014

Carneiro, D. G. S., \& Magalhães, C. M. C. (2020). Percepção de idosos urbanos e ribeirinho sobre o processo de envelhecimento. Brazilian Journal of Health Review, 3(2), p. 2263-2277. https://doi.org/10.34119/bjhrv3n2-077

Chu, W., Chang, S. F., Ho, H. Y., \& Lin, H. C. (2019). The Relationship Between Depression and Frailty in Community - Dwelling Older People: A Systematic Review and Meta - Analysis of 84,351 Older Adults. Journal of Nursing Scholarship, 51(5), 547-559. https://doi.org/10.1111/jnu.12501

Costa, C. R. M., Rodrigues, A. S., Santos, N. B., Teixeira, R. C., \& Lira, S. C. S. (2021). Qualidade de vida do idoso ribeirinho da Amazônia. Saúde Coletiva (Barueri), 11(62), 5236-5249. https://doi.org/10.36489/saudecoletiva.2021v11i62p5236-5249

Costa, R. S., Leão, L. F., \& Campos, H. L. M. (2020). Envelhecer na zona rural do interior do estado do Amazonas, desempenho cognitivo, funcionalidade e percepção de saúde: um estudo transversal. Revista Kairós: Gerontologia, 23(1), 83-103. https://doi.org/10.23925/2176901X.2020v23i1 p83-103

Curcio, C. L., Henao, G. M., \& Gomez, F. (2014). Frailty among rural elderly adults. BMC geriatrics, 14(1) 1-9. https://doi.org/10.1186/1471-2318-14-2

Fabrício, D. M., Chagas, M. H. N., \& Diniz, B. S. (2020). Frailty and cognitive decline. Translational Research. 221, 58-64. https://doi.org/10.1016/j.trsl.2020.01.002

Ferrucci, L., Guralnik, J. M., Studenski, S., Fried, L. P., Cutler Jr, G. B., \& Walston, J. D. (2004). Designing randomized, controlled trials aimed at preventing or delaying functional decline and disability in frail, older persons: a consensus report. Journal of the American Geriatrics Society, 52(4), 625-634. https://doi.org/10.1111/j.1532-5415.2004.52174.x

Freire Junior, R. C., Fernandes, T. G., Borges, G. F., Guerra, R. O., \& Abreu, D. C. C. A. (2018). Factors associated with low levels of physical activity among elderly residents in a small urban area in the interior of the Brazilian Amazon. Archives of Gerontology and Geriatrics, 75, 37-43. https://doi.org/10.1016/j.archger.2017.11.007

Fried, L. P., Tangen, C. M., Walston, J., Newman, A. B., Hirsch, C., Gottdiener, J., ... \& McBurnie, M. A. (2001). Frailty in older adults: evidence for a phenotype. The Journals of Gerontology Series A: Biological Sciences and Medical Sciences, 56(3), M146-M157. https://doi.org/10.1093/gerona/56.3.m146

Gifford, K. A., Bell, S. P., Liu, D., Neal, J. E., Turchan, M., Shah, A. S., \& Jefferson, A. L. (2019). Frailty Is Related to Subjective Cognitive Decline in Older Women without Dementia. Journal of the American Geriatrics Society, 67(9), 1803-1811. https://doi.org/10.1111/jgs.15972

Gutierrez-Robledo, J. M., \& Ávila-Funes, J. A. (2012). How to include the social factor for determining frailty?. J Frailty Aging, 1(1), 13-17. https://doi.org/10.14283/jfa.2012.3

Jesus, I. T. M., Diniz, M. A. A., Lanzotti, R. B., Orlandi, F. S., Pavarin, S. C. I., \& Zazzetta, M. S. (2018). Fragilidade e qualidade de vida de idosos em contexto de vulnerabilidade social. Texto \& Contexto - Enfermagem, 27(4), e4300016. https://doi.org/10.1590/010407072018004300016

Jesus, I. T. M., Orlandi, A. A. D. S., Grazziano, E. D. S., \& Zazzetta, M. S. (2017). Fragilidade de idosos em vulnerabilidade social. Acta Paulista de Enfermagem, 30(6), 614-620. https://doi.org/10.1590/1982-0194201700088

Llano, P. M. P., Lange, C., Sequeira, C. A. C., Jardim, V. M. R., Castro, D. S. P., \& Santos, F. (2019). Fatores associados à síndrome da fragilidade em idosos rurais. Revista Brasileira de Enfermagem, 72 (Supl. 2), 14-21. http://dx.doi.org/10.1590/0034-7167-2017-0079

Mezuk, B., Lohman, M., Dumenci, L., \& Lapane, K. L. (2013). Are depression and frailty overlapping syndromes in mid-and late-life? A latent variable analysis. The American Journal of Geriatric Psychiatry, 21(6), 560-569. https://doi.org/10.1016/j.jagp.2012.12.019

Miyamura, K., Fhon, J. R. S., Bueno, A. A., Fuentes-Neira, W. L., Silveira, R. C. C. P., \& Rodrigues, R. A. P. (2019). Síndrome da fragilidade e comprometimento cognitivo em idosos: revisão sistemática da literatura. Revista Latino-Americana de Enfermagem, 27, e3202. https://doi.org/10.1590/1518-8345.3189.3202

Nascimento, P. P. P. D., \& Batistoni, S. S. T. (2019). Depression and frailty in old age: a narrative review of the literature published between 2008 and 2018. Interface-Comunicação, Saúde, Educação, 23, e180609. https://doi.org/10.1590/Interface.180609 
Nascimento, R. G., Cardoso, R. O., Santos, Z. N. L., Pinto, D. S., \& Magalhães, C. M. C. (2017). Housing conditions and the degree of home satisfaction of elderly riverside residents of the Amazon region. Psico-USF, 22(3), 389-399. https://doi.org/10.1590/1413-82712017220301

Nascimento, R. G., Cardoso, R. O., Santos, Z. N. L., Pinto, D. S., \& Magalhães, C. M. C. (2016). Percepção de idosos ribeirinhos amazônicos sobre o processo de envelhecimento: o saber empírico que vem dos rios. Revista Brasileira de Geriatria e Gerontologia, 19(3), 429-440. http://dx.doi.org/10.1590/1809-98232016019.150121

Neri, A. L., Yassuda, M. S., Araújo, L. F. D., Eulálio, M. D. C., Cabral, B. E., Siqueira, M. E. C. D., ... \& Moura, J. G. D. A. (2013). Metodologia e perfil sociodemográfico, cognitivo e de fragilidade de idosos comunitários de sete cidades brasileiras: Estudo FIBRA. Cadernos de Saúde Pública, 29, 778792. https://doi.org/10.1590/S0102-311X2013000400015

Oliveira, H. G. A., Pereira, M. S., Prestes, Y. A., Silva, E. S., \& Campos, H. L. M. (2020). Características cognitivas e domínio físico funcional em idosos avaliados em domicílio numa cidade no interior do Amazonas: estudo transversal. Revista Kairós: Gerontologia, 23(1), 161-179. https://doi.org/10.23925/2176-901X.2020v23i1p161-179

Ofori-Asenso, R., Chin, K. L., Mazidi, M., Zomer, E., Ilomaki, J., Zullo, A. R., ... \& Bell, J. S. (2019). Global incidence of frailty and prefrailty among community-dwelling older adults: a systematic review and meta-analysis. JAMA network open, 2(8), e198398-e198398. https://doi.org/10.1001/jamanetworkopen.2019.8398

Polaro, S. H. I., Gonçalves, L. H. K., Franhani, C. S., \& Feitosa, E. S. (2013). Condições de vida e saúde de idosos residentes em áreas ribeirinhas. Revista de Enfermagem UFPE, 7(9), 5510-7.

Preto, L. S. R., Conceição, M. D. C. D. D., Amaral, S. I. S., Figueiredo, T. M., \& Preto, P. M. B. (2018). Fragilidade e fatores de risco associados em pessoas idosas independentes residentes em meio rural. Revista de Enfermagem Referência, (16), 73-84. https://doi.org/10.12707/RIV17078

Rauchbach, R., de Souza Wendling, N. M., Scorsato, A. P., Reis, J. C. F., Cortez, A. C. L., \& Dantas, E. H. M. (2019). Reprodutibilidade, objetividade e validade do instrumento de avaliação do nível de atividade física de idosos-Curitibativa (INAFI). Fisioterapia Brasil, 20(3), 317-328. https://doi.org/10.33233/fb.v20i3.2648

Reis Junior, W. M., Carneiro, J. A. O., Coqueiro, R.S., Santos, K. T., \& Fernandes, M. H. (2014). Préfragilidade e fragilidade de idosos residentes em município com baixo Índice de Desenvolvimento Humano. Revista Latino-Americana de Enfermagem, 22(4), 654-661. https://doi.org/10.1590/0104-1169.3538.2464

Ribeiro, E. A. M., Ribeiro, E. E., Viegas, K., Teixeira, F., Santos Montagner, G. F. F., Mota, K. M., ... \& de Paz, J. A. (2013). Functional, balance and health determinants of falls in a free living community Amazon riparian elderly. Archives of gerontology and geriatrics, 56(2), 350-357. https://doi.org/10.1016/j.archger.2012.08.015

Robertson, D. A., Savva, G. M., Coen, R. F., \& Kenny, R. A. (2014). Cognitive function in the prefrailty and frailty syndrome. Journal of the American Geriatrics Society, 62(11), 2118-2124 https://doi.org/10.1111/jgs.13111

Silva, S. S. D. C., Santos, T. M. D., Pontes, F. A. R., \& Maluschke, J. B. (2011). Avaliação de famílias Ribeirinhas: uma proposta adaptada ao contexto. Gerais: Revista Interinstitucional de Psicologia, 4(2), 253-263.

Soares, P. A. O., Ramos, E. R., \& Pinto, E. R. A. (2018). Nível de fragilidade em idosos do município de Alcobaça, BA. Revista Mosaicum, 27, 209-222. https://doi.org/10.26893/rm.v15i27.63

Stoppe Júnior, A., Jacob Filho, W., \& Louza Neto, M. R. (1994). Avaliação de depressão em idosos através da "Escala de Depressão em Geriatria": resultados preliminares. Rev. ABP-APAL, 149 53.

Tavares, D. M. S., dos Santos Ferreira, P. C., Dias, F. A. D., de Morais Souza, L., Gonçalves, J. R. L., \& Rodrigues, L. R. (2017). Aspectos sociodemográficos e desempenho cognitivo de idosos residentes na zona rural. Avances en Enfermería, 35(3), 275-283. https://doi.org/10.15446/av.enferm.v35n3.61789

Vaughan, L., Corbin, A. L., \& Goveas, J. S. (2015). Depression and frailty in later life: a systematic review. Clinical interventions in aging, 10, 1947. https://doi.org/10.2147/cia.s69632

Zazzetta, M. S., Gomes, G. A., Orlandi, F. S., Gratao, A. C., Vasilceac, F. A., Gramani-Say, K., ... \& Nascimento, C. M. (2017). Identifying frailty levels and associated factors in a population living in the context of poverty and social vulnerability. J Frailty Aging, 6(1), 29-32 https://doi.org/10.14283/jfa.2016.116 


\section{CONFLITOS DE INTERESSES}

Não há conflitos de interesses.

\section{FINANCIAMENTO}

Pesquisa financiada pelo Programa de Apoio à Pós-graduação da Coordenação de Aperfeiçoamento de Pessoal de Nível Superior (PROAP/CAPES).

\section{SOBRE OS AUTORES}

Rodolfo Gomes do Nascimento é fisioterapeuta, mestre em Doenças Tropicais pelo Núcleo de Doenças Tropicais da Universidade Federal do Pará (UFPA), doutor e pós-doutorando em Teoria e Pesquisa do Comportamento pela (UFPA). É professor da Universidade Estadual do Pará (UEPA) Universidade da Amazônia (UNAMA) e do Programa de Pós-graduação em Segurança Pública da Universidade Federal do Pará (PPGSP/UFPA).

e-mail: rodgn@hotmail.com

(3) https://orcid.org/0000-0002-4619-5646

Denise da Silva Pinto é fisioterapeuta, mestre em Epidemiologia pela Universidade Federal de São Paulo (UNIFESP), doutora em Doenças Tropicais pelo Núcleo de Medicina Tropical da Universidade Federal do Pará (UFPA). É professora da Faculdade de Fisioterapia e Terapia Ocupacional da UFPA. e-mail: denisefisio23@gmail.com

(3) https://orcid.org/0000-0003-4940-8114

Celina Maria Colino Magalhães é psicóloga, mestre em Teoria e Pesquisa do Comportamento pela (UFPA) e doutora em Psicologia pela Universidade de São Paulo (USP). É professora do Programa de Pós-Graduação em Teoria e Pesquisa do Comportamento da Universidade Federal do Pará, Bolsista de produtividade em Pesquisa 1D-CNPq.

e-mail: celinaufpa@gmail.com

(3) https://orcid.org/0000-0002-1279-179X

Ronald de Oliveira Cardoso é terapeuta ocupacional pela Universidade Estadual do Pará UEPA) e especialista em Saúde da Família pela UEPA.

e-mail: ronald.cardoso01@hotmail.com

(2) https://orcid.org/0000-0001-5938-4676

Katiane da Costa Cunha é fisioterapeuta, mestre em Desenvolvimento e Meio Ambiente Urbano pela Universidade da Amazônia (UNAMA), doutora e pós-doutora em Teoria e Pesquisa do Comportamento pela (UFPA). É professora da Universidade Estadual do Pará (UEPA) e do Programa de Pós-graduação em Segurança Pública da Universidade Federal do Pará (PPGSP/UFPA).

e-mail: katiane.cunha@uepa.br

(2) https://orcid.org/0000-0001-5361-5090

Anna Beatriz de Souza Piedade é graduanda do curso de Fisioterapia da Universidade da Amazônia (UNAMA), Ananindeua-Pará, Brasil.

e-mail: annazbeatriz18@gmail.com

(2) https://orcid.org/0000-0003-0670-7632

Giovana Rodrigues Puga é graduanda do curso de Fisioterapia da Universidade da Amazônia (UNAMA), Ananindeua-Pará, Brasil.

e-mail: giovanapuga0707@gmail.com

(2) https://orcid.org/0000-0003-3203-6000 